\title{
The Misorientation Distribution Function
}

\author{
J. POSPIECH†, K. SZTWIERTNIAT and F. HAESSNER $\ddagger$ \\ tInstytut Podstaw Metallrugii, Krakow, Poland \\ $\ddagger$ Institut für Werkstoffe, Technische Universität, Braunschweig, Germany
}

(Received May 3, 1983; in final form June 17, 1983)§

The analysis of misorientations has up to now usually be carried out by comparing the values obtained experimentally using two dimensional distributions of rotation axes or rotation angles with the distribution calculated by Mackenzie for the statistically random case. In this paper the presentation of the distribution of the misorientations is based on the three dimensional orientation distribution function (ODF) (as described by Bunge). The new function is termed the misorientation distribution function (MDF) to differentiate it from the ODF. The advantages in using this function are presented and illustrated by three MDF's derived from the work of Haessner, Pospiech and Sztwiertnia.

\section{INTRODUCTION}

In many investigations of polycrystalline materials, an important source of information is the orientation differences ("misorientations") between adjacent crystallites as well as the statistics based on them. This information can be derived from the results of measurements of orientation topography (Haessner, 1981).

The misorientations are described statistically using the threedimensional misorientation distribution function (MDF). This function can be described using different orientation parameters. The parameters chosen depend on the nature of the question which one wants to answer. In practice two uncorrelated statistics describing, for instance, the twodimensional distribution of rotation axes and the one-dimensional distribution of rotation angles, which have been derived from mis-

§The delay in publication of this paper was caused by an administrative oversight in the printing. The journal's aim is to publish papers within six months, or less, of their receipt in final form. 
orientation sets, have usually been chosen. These distributions are not constant but vary with the rotation angle, when the misorientation distribution is random. The forms, calculated for cubic symmetry are known from the earlier work of Mackenzie and Thomson (1957) and Mackenzie (1964). They can serve as a reference for identification of rotation axes or rotation angles. Examples of such a procedure are presented in the publications by Aust and Rutter $(1960,1962)$, Ibe (1965) and Ibe and Lücke (1972) as well as Schrode (1977). Here the distributions of rotation axes and rotation angles of recrystallized grains which grow rapidly into the surrounding matrix on annealing are analysed for lead, aluminium, iron-silicon and niobium. Such distributions have been used by Schnell (1974) and Schnell and Grewe (1978) in investigations into the problem of the formation and the structure of high angle boundaries in heavily deformed copper single crystals and recently also by Haessner et al. (1983) in an analysis of orientation topography of rolled polycrystalline copper.

In the following the MDF derived for the misorientations of adjacent crystallites ("pairs") lying on a line parallel to the main axes of the rolling symmetry i.e. rolling, transverse or normal direction (RD, TD, ND) will be presented. For this, the orientation topography used in the above work of Haessner et al. (1983) was employed.

\section{FUNDAMENTAL RELATIONSHIPS}

An obvious way to describe the orientation of a crystallite $A$ (orientation $g_{A}$ ) with respect to the crystallite $B$ (orientation $g_{B}$ ), i.e. the orientation difference $\Gamma=g_{B}^{-1} g_{A}$ is given in terms of the Euler angles $\varphi_{1}, \Phi, \varphi_{2}$. Another convenient way of representing the orientation difference is by means of the rotation axis $r$ and the rotation angle $\omega$. In the following we use mainly the latter method.

In the rotation axis and angle description each crystallite is represented by a rectangular reference frame with a common origin. The rotation axis $\mathbf{r}$ has in both reference frames identical spherical coordinates $\vartheta, \psi$ and rotation by $\omega$ about the axis $\mathbf{r}$ brings both frames into coincidence. The definition of the angles $\vartheta, \psi, \omega$ is illustrated in Figure 1. The set of misorientations $\Gamma$ formulates statistically the misorientation distribution function (MDF) $f(\Gamma)$. In the case considered, this function states the probability $d I / I$ of a misorientation within the interval $d \Gamma$ about $\Gamma$, such that 


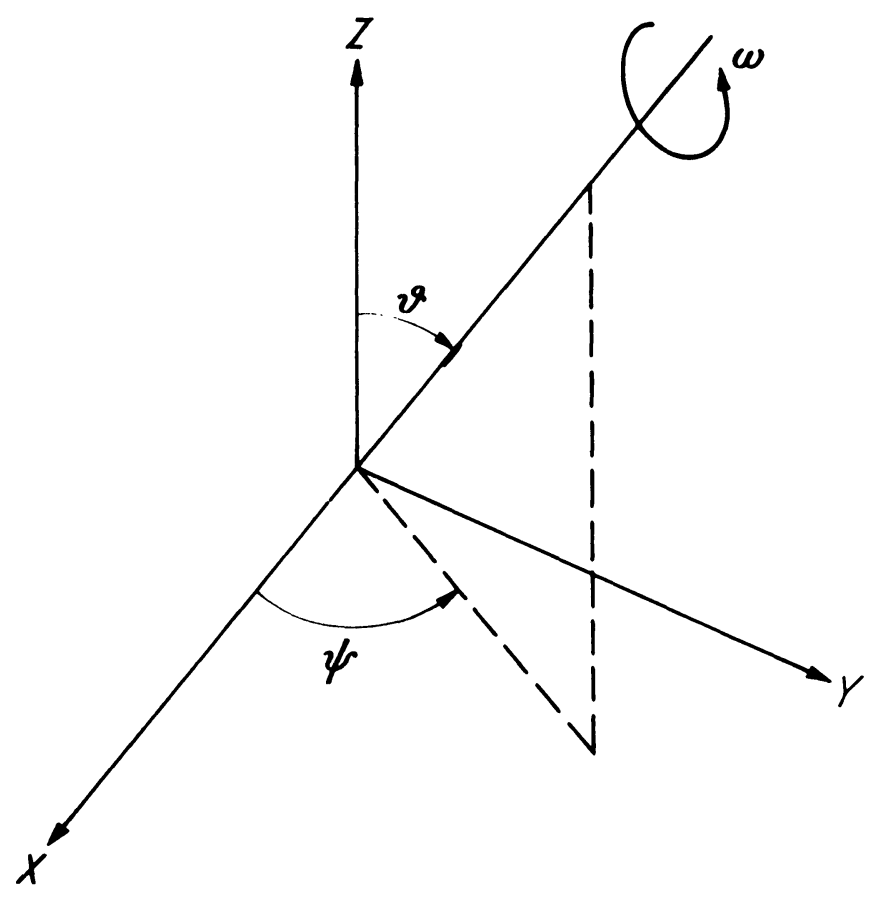

FIGURE 1 Definition of the angles $\vartheta, \psi, \omega$.

$$
\frac{d I}{I}=f(\Gamma(\omega, \mathbf{r})) d \Gamma(\omega, \mathbf{r})
$$

where the element of the space $d \Gamma(\omega, \mathbf{r})$ has the form:

$$
d \Gamma(\omega, \mathbf{r})=\frac{1}{2 \pi^{2}} \sin ^{2} \frac{\omega}{2} \sin \vartheta d \omega \mathrm{d} \vartheta d \psi
$$

Integration of the expression (1) over the rotation angle $\omega$ and division by the element $d s=\sin \vartheta d \vartheta d \psi$ yields the density function of the rotation axis distribution

$$
Q(\mathbf{r})=K^{2} \frac{1}{2 \pi^{2}} \int_{\omega_{0}} f(\omega, \mathbf{r}) \sin ^{2} \frac{\omega}{2} d \omega
$$




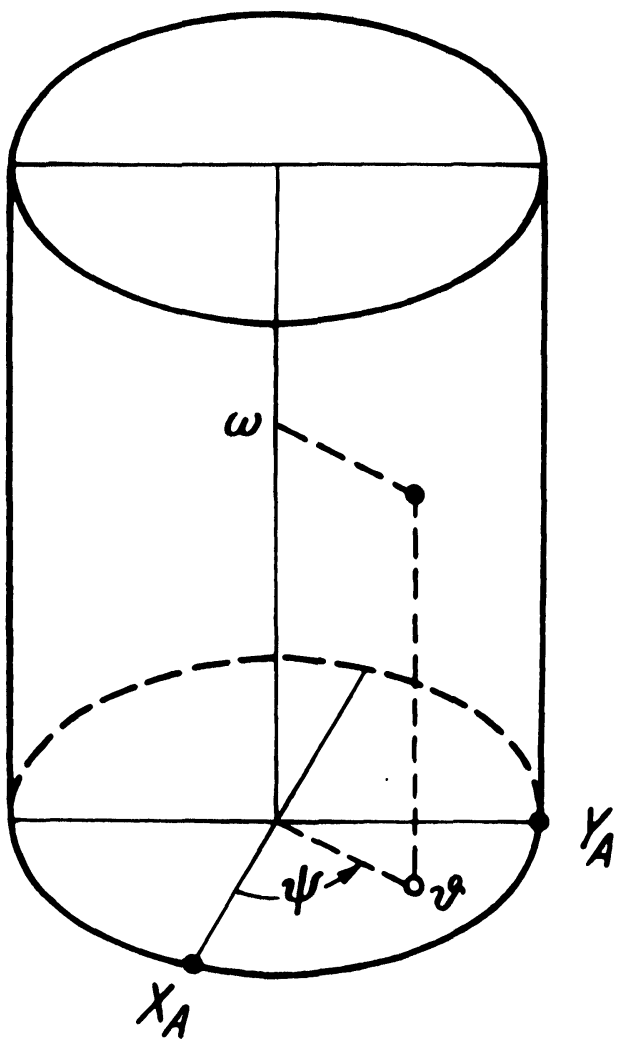

FIGURE 2 Three dimensional representation of the rotation axis $(\vartheta, \psi)$ and rotation angles $(\omega)$.

where $K^{2}$ is a normalizing coefficient. The density function of the rotation angle distribution is determined by integrating the expression (1) over the coordinates of the rotation axes $\vartheta, \psi$ and by dividing by the element $d \omega$

$$
V(\omega)=K^{2} \frac{\sin ^{2} \frac{\omega}{2}}{2 \pi^{2}} \int_{s_{0}} f(\omega, \vartheta, \psi) d s .
$$

The expressions (3) and (4) are integrated within the basic domain of misorientations (see below). The normalizing coefficient $K^{2}$ is equal to 
the number of the basic domains where $K$ is the number of rotation elements in the symmetry group of the crystal. For a random misorientation distribution $(f(\Gamma) \equiv 1)$ and cubic symmetry the density functions $Q(\mathbf{r})$ and $V(\omega)$ have been calculated (Mackenzie and Thomson, 1957, Mackenzie, 1964).

To present the MDF in the $\omega, \vartheta, \psi$-space one can assume a cylindrically shaped region (Figure 2 ) in which the position of the rotation axis $\vartheta, \psi$ is described in a stereographic projection of the basal plane and the rotation angle $\omega$ about an axis perpendicular to this plane. The

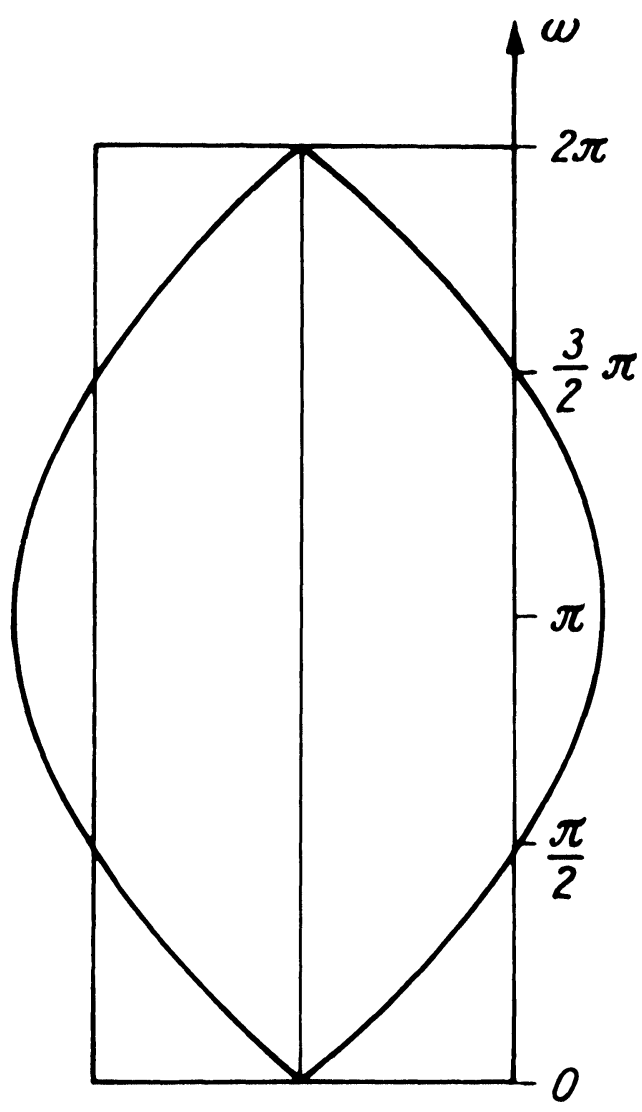

FIGURE 3 Longitudinal section through the cylindrical representation space $(\vartheta, \psi, \omega)$ with details of the deformation of the space. 
form of expression (2) of the element $d \Gamma(\omega, \mathbf{r})$ indicates that the measure

$$
\left(J=\frac{1}{2 \pi^{2}} \cdot \sin ^{2} \frac{\omega}{2} \cdot \sin \vartheta\right)
$$

of this space is not constant.

This fact is illustrated in Figure 3, (Pospiech, 1972), in which the deformation of the longitudinal section of the cylinder, resulting from the linearisation of the measure of the space can be seen. The largest deformation corresponds to the smallest rotation angles $\omega$. For $\omega=0$ the cross section is reduced to a point, which is a manifestation of the fact that each position of a point in this section describes the same (zero) orientation difference.

The crystal symmetry introduces a division of the space into symmetrically equivalent basic domains, i.e. the smallest sub-domains of the space in which all values of the MDF are contained. This division is determined by the condition $f(g)=f\left(c_{j} \cdot g \cdot c_{k}\right)$, where $c_{j}, c_{k}$ are rotation elements of the crystal symmetry.

The cubic symmetry contains 24 rotation elements $c_{i}(i=1,2, \ldots 24)$ and in this case the space is divided into $24 \times 24=576$ basis domains. Thus each misorientation is represented by a set at 576 symmetrical equivalent misorientations $\Gamma_{j k}=c_{j} \Gamma \cdot c_{k}$ and among them (determined by expression $\Gamma_{j}=c_{j} \cdot \Gamma \cdot c_{j}^{-1}$ or $\Gamma_{k}=c_{k} \Gamma c_{k}^{-1}$ ) there are at most 24 misorientations, differing by the rotation angles $\omega$. The smallest of these angles $\left(\omega_{\min }\right)$ is called the angle of misorientation and the rotation axis corresponding to it is called the axis of misorientation. The basic domain of misorientation is chosen for the representation of the MDF.

$\operatorname{MDF} f(\Gamma)$ is obtained using the calculation operations of the Bunge method (Bunge, 1982) based on the series expansion

$$
f(\Gamma)=\sum_{l} \sum_{n, m} C_{l}^{m n} \ddot{T}_{l}^{m n}(\Gamma)
$$

in terms of generalized spherical functions $\ddot{T}_{l}^{m n}(\Gamma)$, which are invariant with regard to the crystal symmetry. The sum over $l$ includes all positive integers (even and odd 1 terms). The coefficients $C_{l}^{m n}$ are calculated from

$$
c_{l}^{m n}=\frac{1}{N} \frac{\exp \left(-l^{2} \varepsilon_{0}^{2} / 4\right)-\exp \left(-(l+1)^{2} \cdot \varepsilon_{0}^{2} / 4\right)}{1-\exp \left(-\varepsilon_{0}^{2} / 4\right)} \sum_{i=1}^{N} \ddot{\ddot{T}}_{l}^{m n}\left(\Gamma_{i}\right)
$$

used for a set of single orientations (Pospiech and Lücke, 1975), where 
$\Gamma_{i}(i=1,2, \ldots N)$ denote misorientations and $\varepsilon_{0}$ is the range of scatter of the $\Gamma_{i}$ assumed depending on the accuracy of measurement.

Routine MDF calculations are made using Euler angles $\varphi_{1}, \Phi, \varphi_{2}$ as orientation parameters. In the final calculation step this function is transformed into the space of the rotation parameters $(\omega, \vartheta, \psi)$ according to the rule given in a paper of Pospiech (1972).

\section{Results}

MDF was determined on the basis of orientation topography in rolled copper (95\% reduction) taken from the work by Haessner et al. (1983). The orientation data for this topography were gained at predetermined sites, using selected area diffraction in transmission electron microscopy for two sections perpendicular to the rolling direction (RD) and to the transverse direction (TD) of the material. The points investigated form a rectangular grid. The separation between two points parallel to RD was $1 \mu \mathrm{m}$, those parallel to ND were separated by $0.5 \mu \mathrm{m}$. The calculation was carried out for three sets of orientation differences which were determined from the orientations of adjacent crystallites ("pairs") along the rolling direction, along the transverse direction and along the normal direction of the sample.

The single misorientations $\Gamma_{i}$ are shown in Figures 4-6 for the sets RD, TD and ND respectively in the space of the rotation parameters $\omega, \vartheta, \psi$. For $\omega=50^{\circ}, 55^{\circ}$ resp. $60^{\circ}$ the basic domain is limited to $\vartheta>27.35^{\circ}, 37.28^{\circ}$ resp. $45^{\circ}$. The basic domain reproduces itself for $\omega$-values $>62.8^{\circ}$ (Mackenzie, 1958). Therefore the representation has been limited up to $\omega=60^{\circ}$. In Figures 4-6 the rotation axes are plotted in a right-hand system. Since it is impossible to distinguish between a right- and a left-hand system each point in Figures 4-6 must be doubled by reflecting at the $\psi=45^{\circ}$ line.

From the double points only those misorientations were considered for the MDF calculation for which $\omega_{\min }$ was greater than $15^{\circ}$. The reason was, that for the lower range of $\omega$ values the resolving power was too small and thus the effect of overlapping of the scattering of symmetrically equivalent positions was observed. In the geometrical centre of these positions, i.e. at the point at which $\omega=0$, the overlapping is at a maximum. The number of misorientations used for the MDF calculation (pairs with $\omega_{\min }>15^{\circ}$ ) were in the particular sets as follows: for RD-28, for TD-54 and for ND-131. 

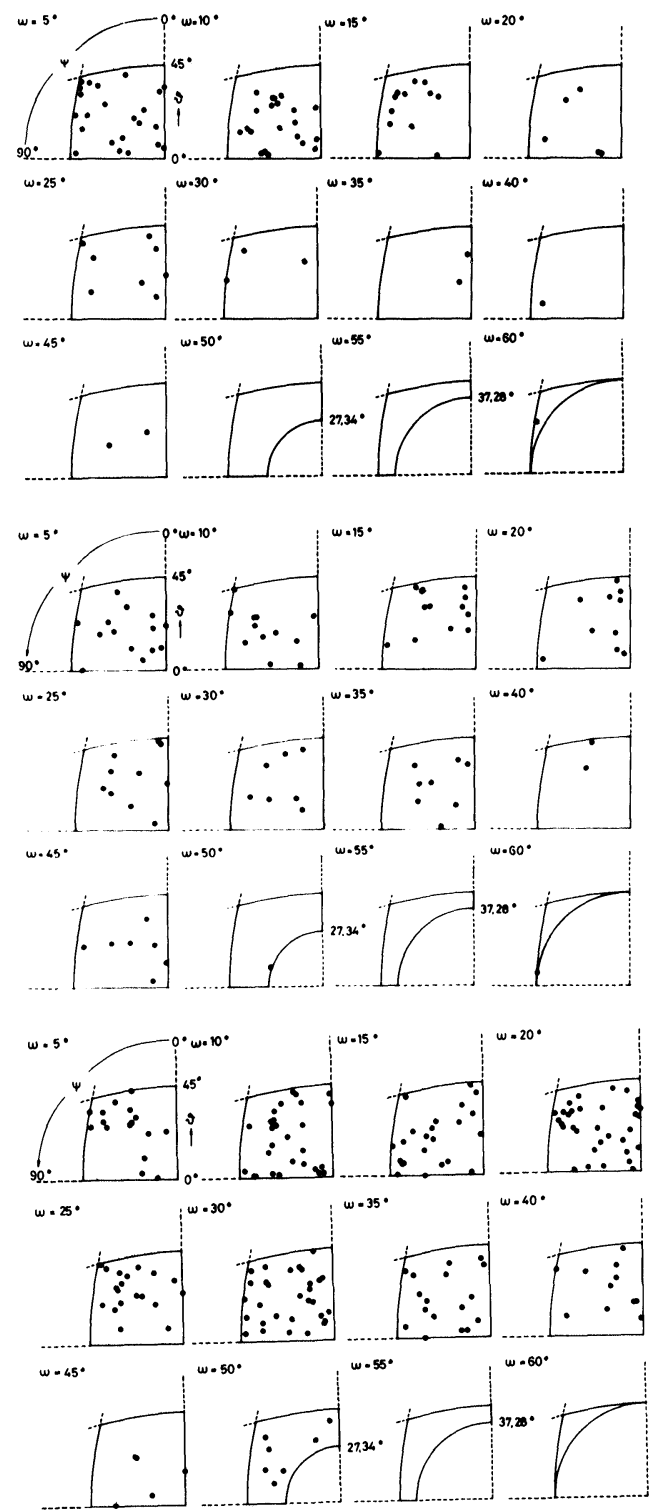

FIGURES 4-6 Individual values of the misorientations $\Gamma_{i}$ in space of the rotation parameters $\vartheta, \omega$ for the set RD (Figure 4), TD (Figure 5) and ND (Figure 6). (The basic domain is limited by the solid line. The intervals are $0 \leqq \omega<7.5^{\circ} ; 7.5^{\circ} \leqq \omega<12.5^{\circ}$; $\left.\ldots, 57.5^{\circ} \leqq \omega\right)$. 

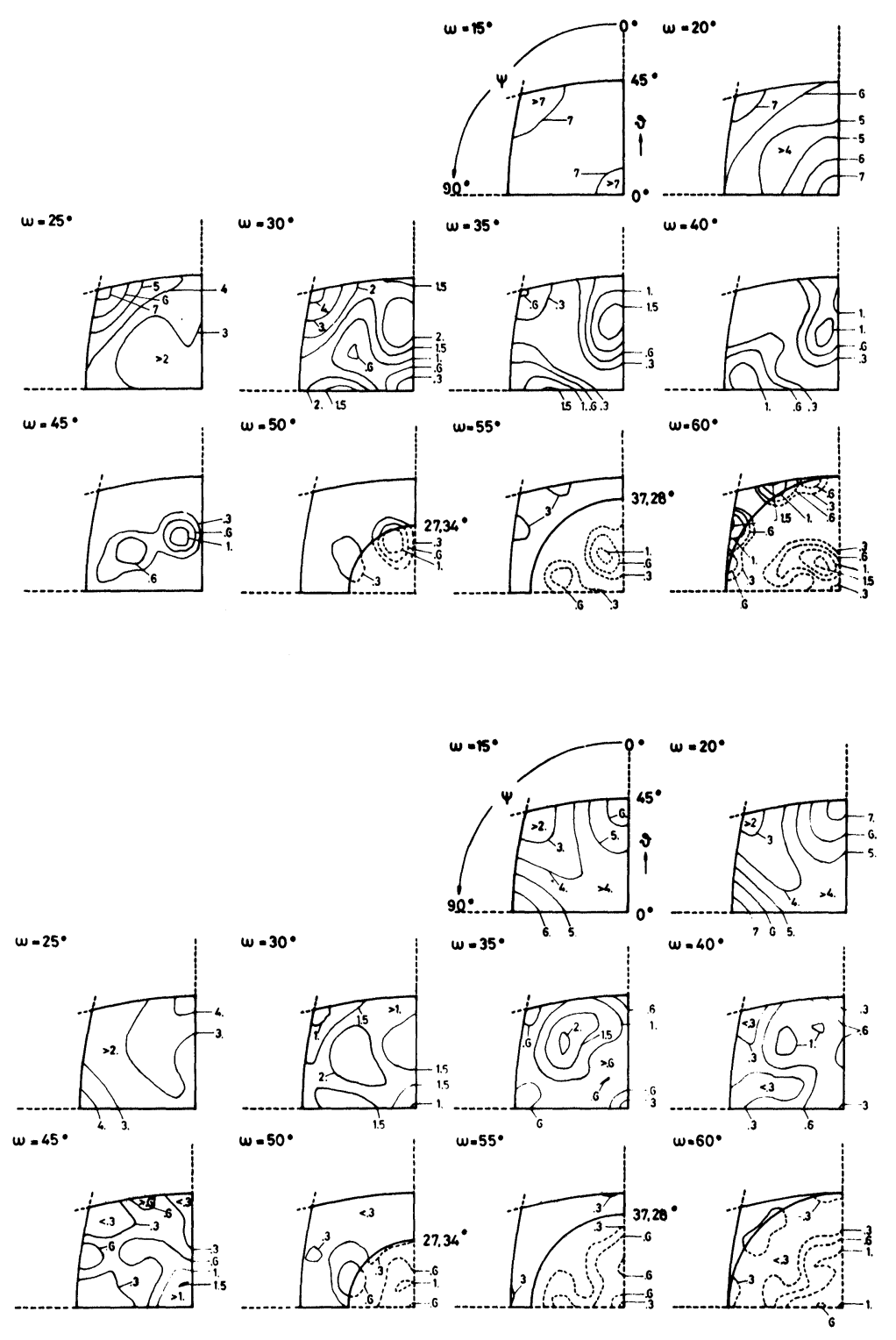


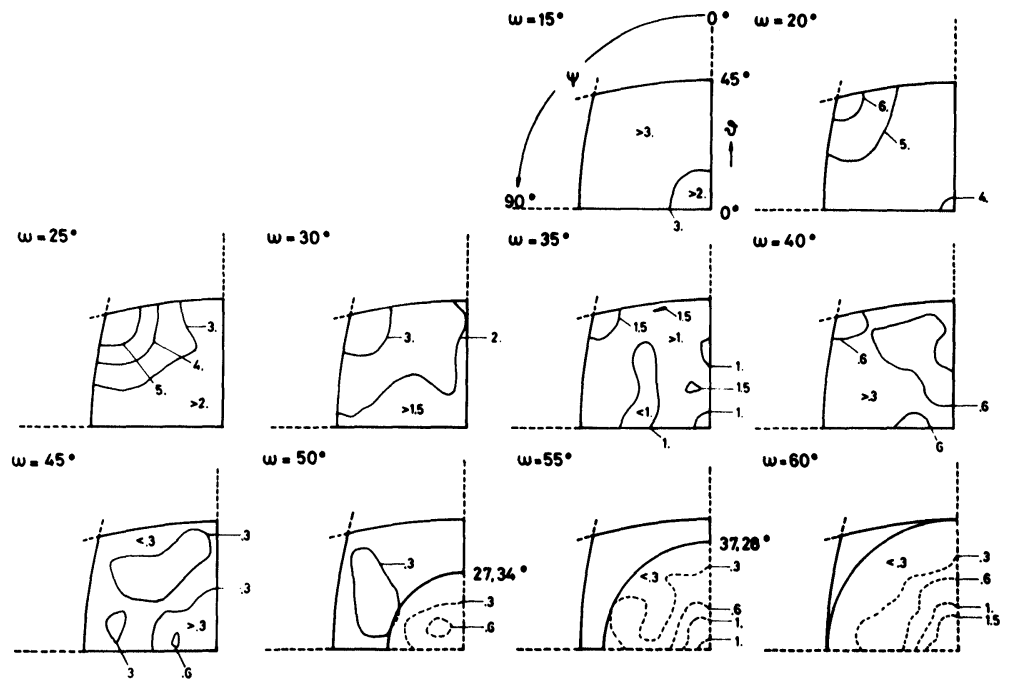

FIGURES 7-9 Misorientation distribution function (MDF) in rotation parameter space for the set RD (Figure 7), TD (Figure 8) and ND (Figure 9). (The basic domain is limited by the solid line).

In the space of the rotation parameters the three MDF's describing the distribution of misorientations for the sets RD, TD, and ND, respectively, are shown in Figures 7-9. If the individual misorientations $\Gamma_{i}$ in Figures 4-6 are compared with the corresponding MDF's in Figures 7-9 then it becomes apparent that for certain angles $\omega$ in the MDF, intensity values are given, although no single misorientation exists for these angles (e.g. set $\mathrm{RD}: \omega=50^{\circ}$ or $55^{\circ}$ ). This is due to the interpolation effect, which is caused by the series expansion (5). The same facts as in Figures 7-9 are represented in the Euler-space by Figures 10-12. Since the space reproduces itself for $\varphi_{2}>45^{\circ}$ the representation has been limited to the interval $0^{\circ} \leqq \varphi_{2} \leqq 45^{\circ}$. Figures 13-15 show regional enlargements from Figures 10-12 for the $\varphi_{2}$-values $40^{\circ}$ (set RD), $5^{\circ}$ (set TD) and $40^{\circ}$ (ND). In these enlargements the same intensity levels have been used as in Figures 10-12.

Consider first the results in the space of the rotation parameters: The distributions obtained are characterized by large areas of scatter. Within them there appear weak maxima which are merely an indication of the tendency to favour the symmetry axes. Thus in the distribution of the misorientation along RD a weak maximum in the position of the $\langle 111\rangle$ axis within the whole range of the rotation angles can be distinguished. 

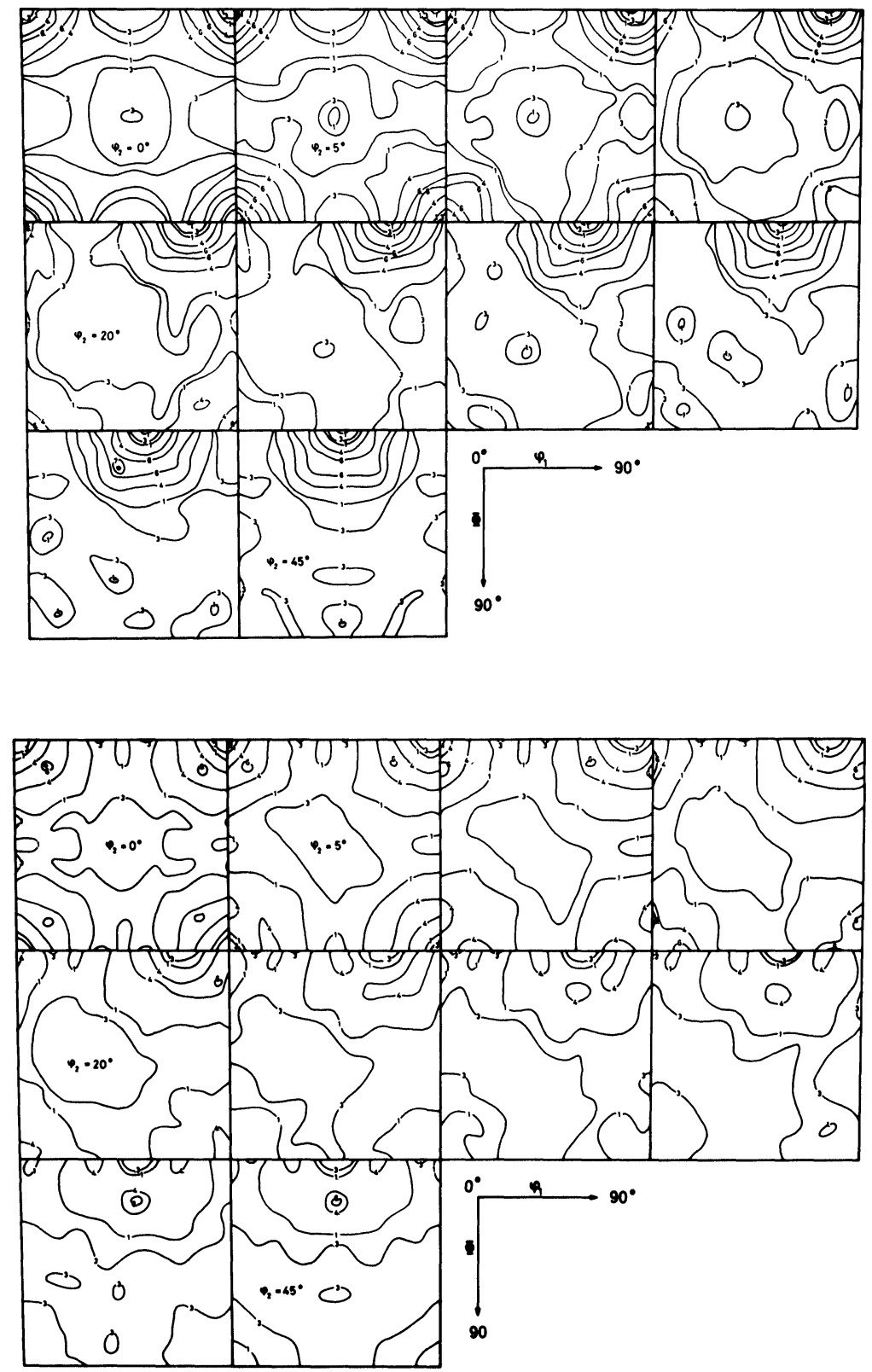


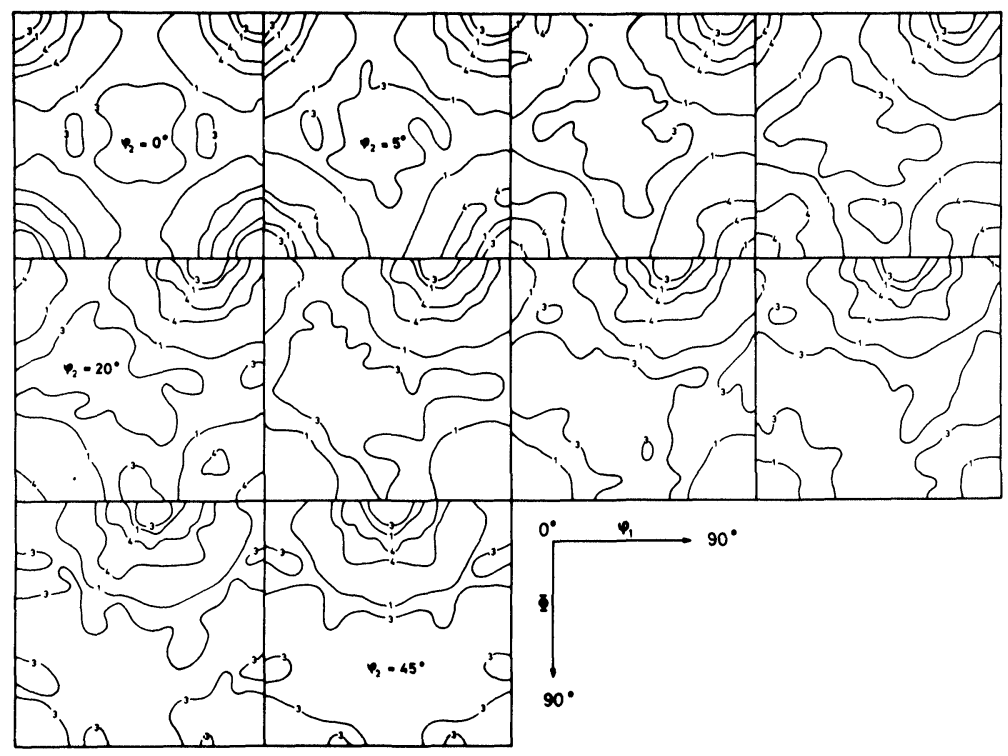

FIGURE 10-12 Misorientation distribution function (MDF) in Euler-space $\left(\varphi_{1}, \phi, \varphi_{2}\right)$ for the set RD (Figure 10), TD (Figure 11) and ND (Figure 12).

At the same time, there occurs a weak concentration at $\langle 100\rangle$ within the range of rotation angles $\omega \sim 15 \div 20^{\circ}$. In the distribution along TD we observe a maximum in the scattering at $\langle 110\rangle$ of 7.4 in the range of rotation angles $\omega \sim 15 \div 20^{\circ}$, and within a weak maximum at $\langle 112\rangle$ $\omega \sim 30 \div 40^{\circ}$. Finally, in the distribution along ND, a maximum of 6 occurs near to $\omega=25^{\circ}$ for $\langle 111\rangle$ similar to the case of RD.

The maxima described above are only obvious after careful study in the Euler-space. The maximum in the set ND lies at the Euler-angles $\varphi_{1}=33.75^{\circ} \Phi=20.42^{\circ}$ and $\varphi_{2}=7.75^{\circ}$ (compare Figure 12 and Figure 15); the maximum in the set TD at $\langle 110\rangle, \omega \simeq 20^{\circ}$ is found at the Eulerangles $\varphi_{1}=7.1^{\circ} \Phi=14.1^{\circ} \varphi_{2}=7.1^{\circ}$ (compare Figure 11 and Figure 14). The minima in the distribution in the Euler-space, for example along the line $\Phi=0$ are most obvious. In the present case the picture of the MDF in the Euler-space is more complex than in the space of the rotation parameter.

Finally the advantages and disadvantages of the two spaces to calculate and represent MDF should be presented. The advantages of the rotation axis space are as follows: 
$t$ is possible to differentiate between exactly determinable "accurate" orientations and inexactly determinable "inaccurate" misorienta1s. The MDF picture is relatively clear. The disadvantages of the rota-
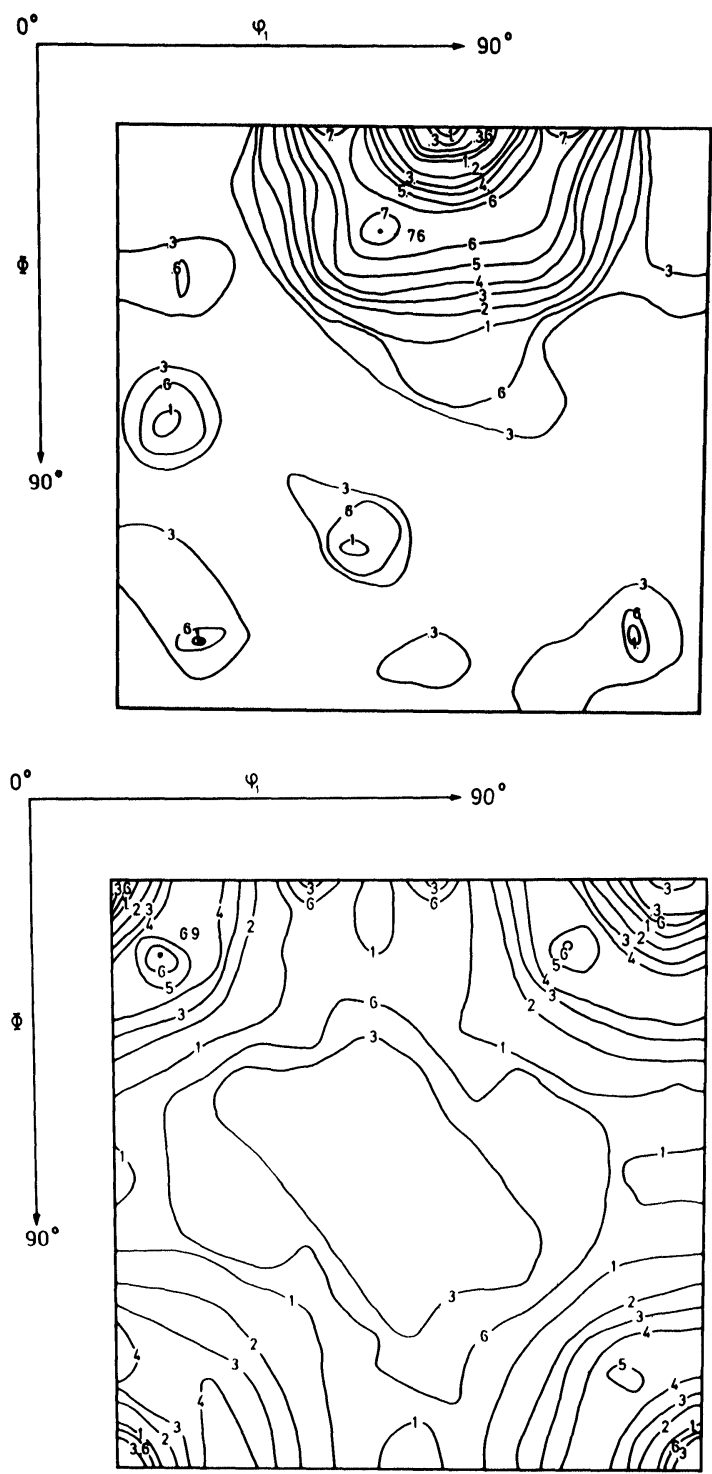


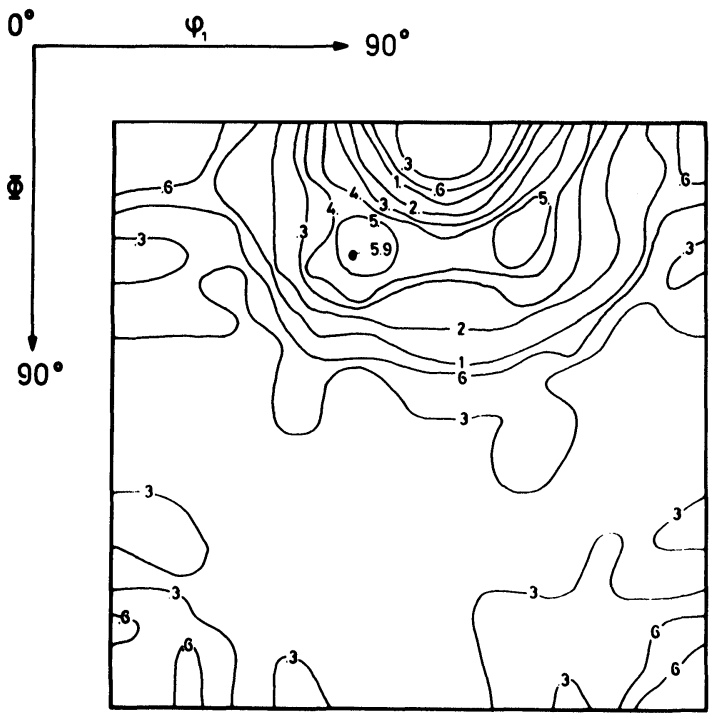

FIGURE 13 Detail enlargement of the MDF in Euler-space for $\varphi_{2}=40^{\circ}$ (set RD; Figure 13)

tion axis space are: numerical calculations are difficult. The space for small $\omega$ is very distorted. The limitation of the basal region is complicated. The evaluation for the Euler-space is practically the reverse. The advantages of the rotation space are here the disadvantages and viceversa. Routine numerical calculations can be carried out in Euler-space.

\section{Acknowledgement}

This work has been supported by the Deutsche Forschungsgemeinschaft.

\section{References}

Aust, K. T. and Rutter, J. W. Trans. Met. Soc. AIME 21850 (1960); 224111 (1962).

Bunge, H. J. Texture Analysis in Materials Science, Butterworths, London (1982).

Haessner, F. Orientation Topography and its Analysis in Rolled Material, Proc. 6th Int.

Conf. on Textures of Materials, Tokyo. Ed. S. I. Nagashima, Iron and Steel Institute of Japan, Tokyo, Vol. I, p. 223 (1981).

Haessner, F., Pospiech, J. and Sztwiertnia, K. Mat Sci. and Engineering, 1 (1983).

Ibe, G. Doctoral Thesis, Rheinisch-Westfälische Technische Hochschule, Aachen (1965). Ibe, G. and Lücke, K. Texture 1, 87 (1972).

Mackenzie, J. K. and Thompson, M. J. Biometrika 44, 205 (1957).

Mackenzie, J. K. Biometrica 45, 229 (1958). 
Mackenzie, J. K. Acta Met. 12, 223 (1964).

Pospiech, J. Kristall und Technik 7, 1057 (1972).

Pospiech, J. and Lūcke, K. Acta Met 23, 997 (1975).

Schnell, Ch. Doctoral Thesis, Universität Münster (1974).

Schnell, Ch. and Grewe, H. G. Electron Microscopical Studies of Orientation Relationships on Large Angle Grain Boundaries Formed During Cold Work of Copper Single Crystals, Proc. 5th Int. Conf. on Textures of Materials, Aachen. Eds. G. Gottstein and K. Lücke, Springer-Verlag, Berlin, Vol. I, p. 389 (1978).

Schrode, W. Doctoral Thesis, Technische Universität Stuttgard (1977). 